Dermatology 2009;218:385-386

DOI: $\underline{10.1159 / 000202986}$

\section{Oblique View Dermoscopy Changes Regular Fibrillar Pattern into Parallel Furrow Pattern}

Yoshifumi Maumi, Masayuki Kimoto, Ken Kobayashi, Naoko Ito, Toshiaki Saida, Masaru Tanaka

Department of Dermatology, Tokyo Women's Medical University Medical Center East, Tokyo, and Shinshu University School of Medicine, Matsumoto, Japan

\section{Key Words}

Fibrillar pattern $\cdot$ Paralles furrow pattern $\cdot$ Dermoscopy $•$ Oblique view dermoscopy

For the effective early detection of acral melanoma, Saida et al. $[1,2]$ first proposed the $7-\mathrm{mm}$ rule, which recommended the excision and histopathologic evaluation of acquired plantar acral melanocytic nevi with a maximum diameter of more than $7 \mathrm{~mm}$. As they later collected dermoscopic observations showing that the parallel ridge pattern is highly specific to early acral melanoma [3], they revised their guideline so that lesions with marked irregularity or parallel ridge patterns should also be excised, even when they are $7 \mathrm{~mm}$ or less [4]. Recently, they further revised their management strategy for acquired acral melanocytic lesions into a 3-step algorithm [5]. In this article, the fibrillar patterns are first divided into regular fibrillar patterns and irregular fibrillar patterns. Lesions $7 \mathrm{~mm}$ or less without the parallel ridge pattern and lesions larger than $7 \mathrm{~mm}$ showing a typical benign pattern (parallel furrow, lattice-like, or regular fibrillar pattern) should just continue to be observed.
The majority of melanocytic nevi on the soles may show 3 major dermoscopic patterns depending on the anatomical site on the sole [6]: the parallel furrow, the lattice-like, and the fibrillar patterns. Regular or irregular patterns should be identified in the fibrillar pattern. The regular fibrillar pattern is seen in acral volar melanocytic nevus, whereas the irregular fibrillar pattern is often seen partly or entirely in acral lentiginous melanoma of the pressure-loaded area of the sole. However, the difference between regular and irregular fibrillar patterns has not yet clearly been defined, although it is very important to distinguish these differences to discriminate an early acral melanoma from an acquired acral melanocytic nevus. The regular fibrillar pattern can be regarded as an artifactual expression of the parallel furrow pattern $[5,6]$. We have proved that the fibrillar pattern is directly caused by an oblique arrangement of melanin pigment columns in the slanting stratum corneum with a three-dimensional reconstruction method using specimens of acral nevi of parallel furrow or fibrillar patterns [7]. The slanting of the stratum corneum is considered to be produced by mechanical pressure from the body weight. Miyazaki et al. [6] already reported that the regular fibrillar pattern of an acral nevus was changed to the parallel furrow pattern by horizontally moving the stratum corneum with the probe of a dermoscope. Therefore, oblique view dermoscopy at a angle to the stratum corneum could change the regular fibrillar pattern into a parallel furrow pattern.

A 37-year-old Japanese female presented with a dark brown macule on the lateral side of the left sole (body-weight-loaded area). She had been aware of the lesion for several years. Dermoscopy examination revealed a typical regular fibrillar pattern, and a diagnosis of acquired melanocytic nevus was made. The slanting angle of the melanin columns in the stratum corneum was confirmed by use of DermLite DL-100 (3Gen, Dana Point, Calif., USA). After the direction of viewing was fixed so that the parallel furrow pattern was observed, pictures were taken with the Der-
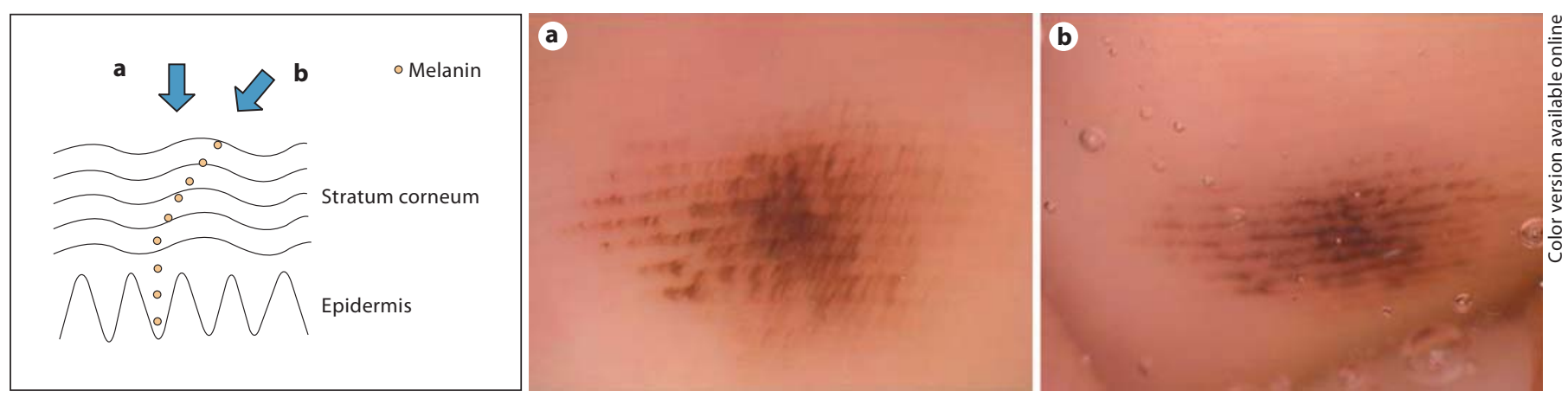

Fig. 1. The ordinary dermoscopy view (a) showed a typical regular fibrillar pattern, whereas the oblique dermoscopy view (b) revealed a parallel furrow pattern. 
ma9500 (Derma Medical, Yokohama, Japan) and K-Y Jelly (Johnson and Johnson, New Brunswick, N.J., USA) (fig. 1).

A picture taken by ordinary dermoscopy (fig. 1a) demonstrated a typical regular fibrillar pattern, but a picture through obliqueview dermoscopy (fig. 1b) showed a parallel furrow pattern and the parallel ridge pattern was not recognized.

To our regret, we have not yet confirmed whether an early acral melanoma with the irregular fibrillar pattern would show the parallel ridge pattern by this new method. If the distinction between regular and irregular fibrillar patterns could be made by these methods, it would be extremely useful for the discrimination of an early acral melanoma from an acral nevus.

However, regarding the photographs from the oblique view, focusing is very difficult because the bubbles in the jelly are seen more clearly due to the space between them. We would like to develop better techniques to obtain a clearer image.

\section{References}

1 Saida T, Ishihara Y, Tokuda Y: Effective detection of plantar malignant melanoma. Int J Dermatol 1993;32:722-725.

2 Saida T, Yoshida N, Ikegawa S, Ishihara K, Nakajima T: Clinical guidelines for the early detection of plantar malignant melanoma. J Am Acad Dermatol 1990;23:37-40.

-3 Oguchi S, Saida T, Koganehira Y, Ohkubo S, Ishihara Y, Kawachi S: Characteristic epiluminescent microscopic features of early malignant melanoma on glabrous skin: a videomicroscopic analysis. Arch Dermatol 1998; $134: 563-568$.

4 Saida T: Malignant melanoma on the sole: how to detect the early lesions efficiently. Pigment Cell Res 2000;13(suppl 8):135-139.

5 Saida T, Koga H: Dermoscopic patterns of acral melanocytic nevi: their variations, changes, and significance. Arch Dermatol 2007;143:14231426.

6 Miyazaki A, Saida T, Koga H, Oguchi S, Suzuki T, Tsuchida T: Anatomical and histopathological correlates of the dermoscopic patterns seen in melanocytic nevi on the sole: a retrospective study. J Am Acad Dermatol 2005;53:230-236

$\checkmark 7$ Kimoto M, Sakamoto M, Iyatomi H, Tanaka M: Three-dimensional melanin distribution of acral melanocytic nevi is reflected in dermoscopy features: analysis of the parallel pattern. Dermatology 2008;216: 205-212.

Yoshifumi Maumi

Department of Dermatology

Tokyo Women's Medical University Medical Center East

Tokyo (Japan)

Tel. +81 33810 1111, Fax +81338941441

E-Mail maujapan2003@ybb.ne.jp 